\title{
Fluorescence Imaging
}

How to cite: Angew. Chem. Int. Ed. 2021, 60, 26685-26693

\section{Targetable Conformationally Restricted Cyanines Enable Photon- Count-Limited Applications**}

\author{
Patrick Eiring ${ }^{+}$, Ryan McLaughlin ${ }^{+}$, Siddharth S. Matikonda ${ }^{+}$Zhongying Han ${ }^{+}$, \\ Lennart Grabenhorst ${ }^{+}$, Dominic A. Helmerich, Mara Meub, Gerti Beliu, Michael Luciano, \\ Venu Bandi, Niels Zijlstra, Zhen-Dan Shi, Sergey G. Tarasov, Rolf Swenson, Philip Tinnefeld, \\ Viktorija Glembockyte,* Thorben Cordes,* Markus Sauer,* and Martin J. Schnermann*
}

\begin{abstract}
Cyanine dyes are exceptionally useful probes for a range of fluorescence-based applications, but their photon output can be limited by trans-to-cis photoisomerization. We recently demonstrated that appending a ring system to the pentamethine cyanine ring system improves the quantum yield and extends the fluorescence lifetime. Here, we report an optimized synthesis of persulfonated variants that enable efficient labeling of nucleic acids and proteins. We demonstrate that a bifunctional sulfonated tertiary amide significantly improves the optical properties of the resulting bioconjugates. These new conformationally restricted cyanines are compared to the parent cyanine derivatives in a range of contexts. These include their use in the plasmonic hotspot of a DNA-nanoantenna, in single-molecule Förster-resonance energy transfer (FRET) applications, far-red fluorescence-lifetime imaging microscopy (FLIM), and single-molecule localization microscopy (SMLM). These efforts define contexts in which eliminating cyanine isomerization provides meaningful benefits to imaging performance.
\end{abstract}

\section{Introduction}

Modern fluorescence-based methods put intense demands on fluorescent probes. For example, single-molecule localization microscopy (SMLM) methods, such as direct stochas- tic optical reconstruction microscopy ( $d$ STORM), depend on the availability of bright photoactivatable or photoswitchable dyes. $^{[1]}$ In these experiments the precision of the singlemolecule localization depends directly on photon count. ${ }^{[1]}$ Despite recent progress in the development of new fluorophores, SMLM and other single-molecule experiments in the far-red are still limited to a few organic fluorophores. In particular, cyanine dyes, especially the pentamethine cyanines Cy5 and Alexa Fluor 647 (Figure 1A), have proven to be invaluable probes in a range of contexts. ${ }^{[2]}$ However, the excited state of these molecules is prone to a deactivating excited-state trans-to-cis photoisomerization that reduces photon emission. ${ }^{[2 c]}$

We recently introduced the novel pentamethine cyanine, Cy5B, where photoisomerization is blocked by installation of a conformationally restraining ring system. ${ }^{[3]}$ Relative to conventional cyanines, these compounds exhibit improved fluorescence quantum yield and longer fluorescence lifetimes. Small molecule conjugates undergo efficient recovery from hydride reduction (reductive caging) to enable high quality SMLM in oxygenated buffer. ${ }^{[3 a, 4]}$ However, immunofluorescent and other biomolecule targeted applications were not possible due to the propensity of these compounds to form non-fluorescent aggregates.
[*] P. Eiring, ${ }^{[+]}$D. A. Helmerich, M. Meub, Dr. G. Beliu,

Prof. Dr. M. Sauer

Department of Biotechnology and Biophysics Biocenter, Julius-

Maximilians-Universität Würzburg

Am Hubland, 97074 Würzburg (Germany)

E-mail:m.sauer@uni-wuerzburg.de

R. McLaughlin, ${ }^{[+]}$Dr. S. S. Matikonda, ${ }^{[+]}$Dr. M. Luciano, Dr. V. Bandi,

Dr. M. J. Schnermann

Laboratory of Chemical Biology, Center for Cancer Research,

National Cancer Institute

Frederick, MD 21702 (USA)

E-mail: martin.schnermann@nih.gov

Z. Han, ${ }^{[+]}$Dr. N. Zijlstra, Prof. Dr. T. Cordes

Physical and Synthetic Biology, Faculty of Biology, Ludwig-Maximilians-Universität München

Großhadernerstr. 2-4, 82152 Planegg-Martinsried (Germany)

E-mail: cordes@bio.Imu.de

L. Grabenhorst, ${ }^{[+]}$Prof. Dr. P. Tinnefeld, Dr. V. Glembockyte Department of Chemistry and Center for NanoScience, LudwigMaximilians-Universität München

Butenandtstr. 5-13, 81377 München (Germany)

E-mail: v.glembockyte@Imu.de
Dr. Z. D. Shi, Dr. R. Swenson

Chemistry and Synthesis Center, National Heart, Lung, and Blood Institute, $\mathrm{NIH}$

Rockville, MD 20850 (USA)

Dr. S. G. Tarasov

Biophysics Resource in the Center for Structural Biology, Center for Cancer Research, National Cancer Institute

Frederick, MD 21702 (USA)

$\left.{ }^{+}\right]$These authors contributed equally to this work.

[**] A previous version of this manuscript has been deposited on a preprint server (https://chemrxiv.org/engage/chemrxiv/articledetails/60c759aa567dfe6399ec6aa9).

(). Supporting information and the ORCID identification number(s) for

(iD the the author(s) of this article can be found under: https://doi.org/10.1002/anie.202109749.

(C) 2021 The Authors. Angewandte Chemie International Edition published by Wiley-VCH GmbH. This is an open access article under the terms of the Creative Commons Attribution License, which permits use, distribution and reproduction in any medium, provided the original work is properly cited. 
A

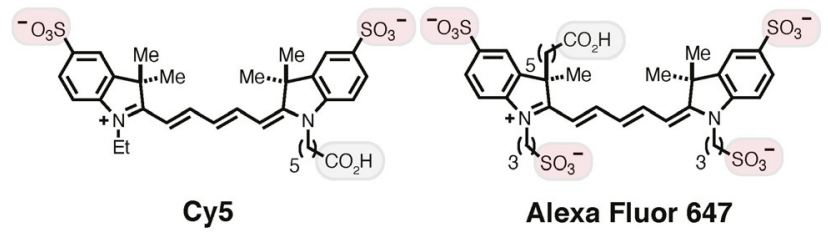

B

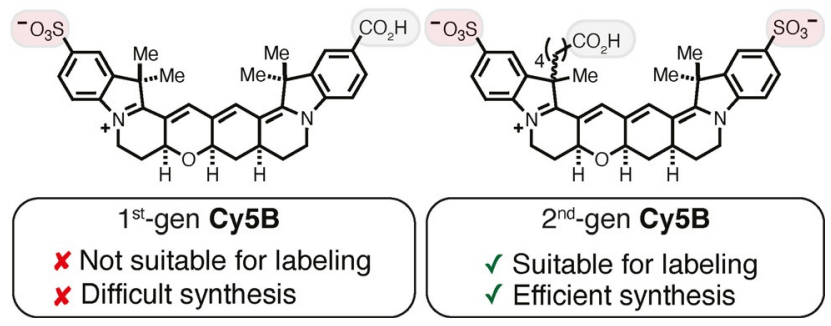

Figure 1. (A) Previously reported pentamethine cyanines, ${ }^{[2 c, 8]}$ and (B) evolution of restrained pentamethine variants.

Here we report the optimized synthesis of di-sulfonated and tri-sulfonated derivatives and detail the application of these probes in several settings. Persulfonation and other charge-optimization strategies have been established to improve probe properties for advanced imaging applications. ${ }^{[2, \mathrm{~d}, 5]}$ We investigate the use of these probes in several challenging contexts, including in the plasmonic hotspot of DNA-nanoantenna, in single-molecule Förster resonance energy transfer (smFRET) based applications, far-red fluorescence lifetime imaging microscopy (FLIM), and SMLM. By benchmarking the restrained sulfonated cyanines against unrestrained cyanines and other commonly used dyes, we establish contexts where reduced polymethine isomerization positively impacts imaging performance.

\section{Results and Discussion}

Scheme 1 details the synthesis of di- and tri-sulfonated conformationally restrained pentamethine cyanines. Indolenine $\mathbf{3}$, bearing a functionalizable methyl ester, was accessed through Fisher indole synthesis and alkylation from 1. Similar to prior efforts, we found that conventional cyanine formation with $\mathbf{3}$ and previously reported $\mathbf{4}$ afforded the desired mixed cyanine in modest yield (14\%) after a complex purification process. $^{[2 \mathrm{~d}]}$ As a consequence, we examined several alternative approaches to unsymmetrical cyanine formation. ${ }^{[6]}$ Notably, solid phase methods proved difficult to implement on significant scale. By contrast, a solution-phase sequential heterocycle addition sequence, based on a report by Wolf and co-workers, provides scalable reproducible access to rac-6 $\mathbf{6}$ on up to gram scales. ${ }^{[6 \mathrm{~d}]}$ This approach involved addition of $\mathbf{4}$ to the cyanine precursor, malonaldehyde bis(phenylimine) monohydrochloride, with microwave irradiation $\left(130^{\circ} \mathrm{C}\right.$, $30 \mathrm{~min}$ ) to first form acylated hemicyanine $\mathbf{5}$. This unpurified intermediate was then reacted with $\mathbf{3}$ at r.t. to provide unsymmetrical pentamethine cyanine rac-6, in good yield (58\% from 3) with only trace formation of other cyanine products. While installation of the $\alpha-\beta$ unsaturated aldehyde via cross-metathesis reaction was unsuccessful initially due to the poor solubility of $\mathbf{3}$ in organic solvent, we ultimately found that the addition of solubilizing counterion, tetrabutylammonium bromide, enabled the reaction to proceed efficiently. The key tetracyclization reaction was carried out using $\mathrm{BBr}_{3}$ in $\mathrm{CH}_{2} \mathrm{Cl}_{2}$, again with tetrabutylammonium bromide acting as a critical solubility-enhancing additive. As observed previously, ${ }^{[3 a]}$ the initial cyclization reaction formed a mixture of diastereomers which were equilibrated using protic acidic conditions to form the syn product rac-7, which is a mixture of diastereomers due to the additional chiral center. The mixture

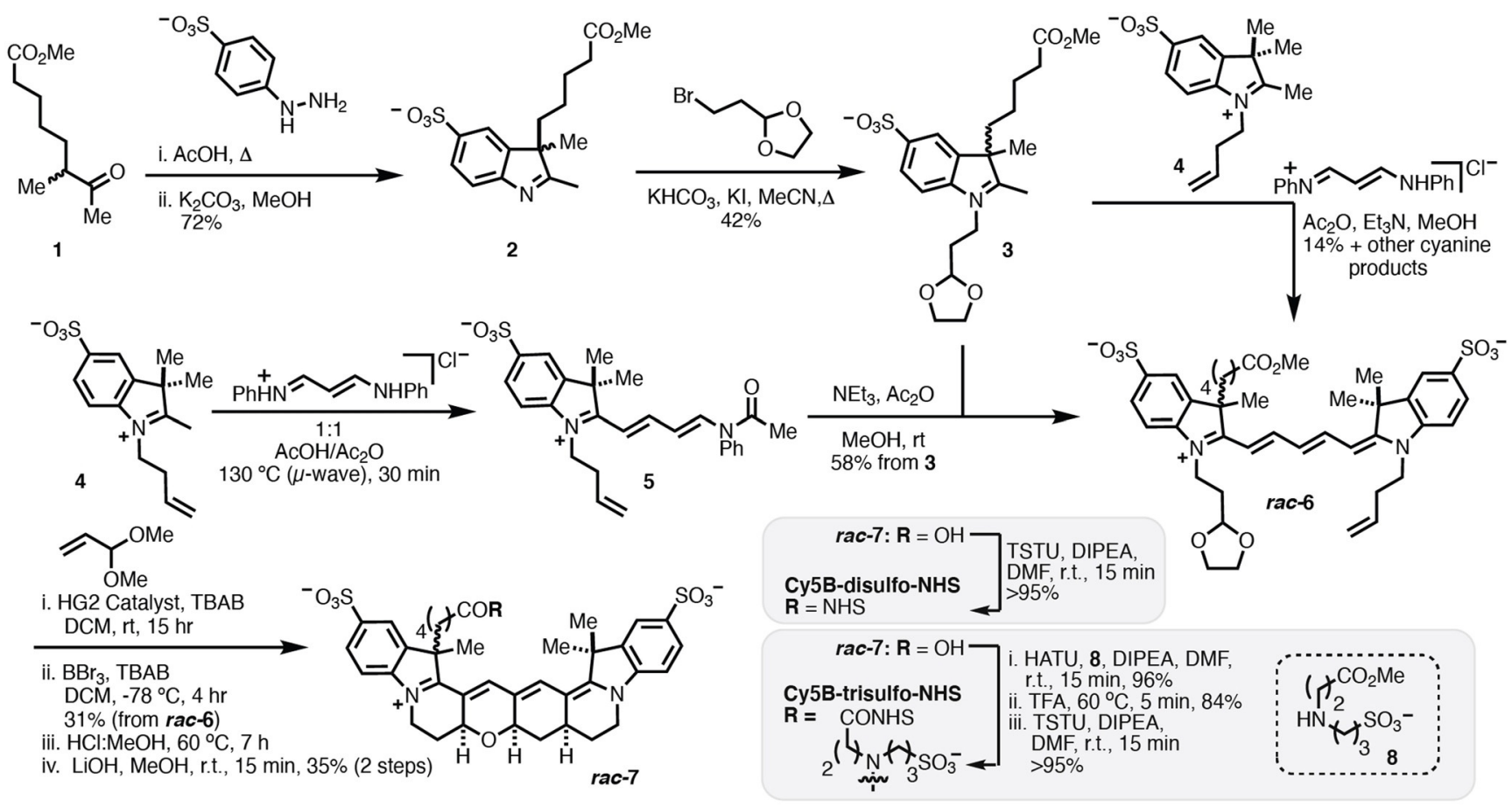

Scheme 1. Synthesis of Cy5B-disulfo and -trisulfo NHS Esters. 
rac-7 could be converted to the corresponding NHS-ester, Cy5B-disulfo-NHS, through a saponification/TSTU sequence. Alternatively, the tertiary amide, Cy5B-trisulfo-NHS, could be formed via saponification and coupling with amine $\mathbf{8}$, followed by saponification/TSTU NHS-ester formation.

We then assessed the optical properties of these compounds, as well as their antibody conjugates (Table 1, Table S1). As a comparison, we used mAb conjugates of Cy5. ${ }^{[7]}$ As free dyes, both conformationally restrained variants Cy5B-disulfo and Cy5B-trisulfo have higher quantum yield $\left(\Phi_{\mathrm{F}}\right)$ and longer fluorescence lifetimes $\left(\tau_{\mathrm{F}}\right)$ than Cy5, albeit with moderately reduced extinction coefficients $(\varepsilon)$ in an aqueous environment (Table 1). These values (in $\mathrm{H}_{2} \mathrm{O}$ ) are quite similar to the monosulfonated derivative we reported previously. ${ }^{[3 a]}$ We then prepared and evaluated the goat antimouse antibodies labeled on average with a degree of labeling (DOL) of 1 and 3 using conventional NHS-ester labeling conditions (Figure S1). Several observations emerge from the spectroscopic properties of the resulting conjugates. First, with unrestrained $\mathrm{Cy} 5, \Phi_{\mathrm{F}}$ decreased substantially, that is, from 0.21 to 0.11 for DOL 3.0 and $\tau_{\mathrm{F}}$ decreased only slightly, which is in line with prior observations (Table 1). ${ }^{[7,8]}$ With the Cy5B-disulfo-mAb conjugates, $\Phi_{\mathrm{F}}$ is similar to the free dye, but moderately reduced (by $31 \%$ ) at higher labeling density. By contrast, the trisulfo-derivative, exhibits nearly identical $\Phi_{\mathrm{F}}$ at both labeling densities, and moderately increased fluorescence lifetimes. These results suggest that this simple amide modification strategy improves the properties of the

Table 1: Spectroscopic characteristics of (A) free dyes and (B) mAb conjugates in PBS, $\mathrm{pH} 7.4$ The extinction coefficients were measured in triplicate ( \pm standard deviation (s.d.)).

\begin{tabular}{|c|c|c|c|c|c|}
\hline \multicolumn{6}{|l|}{$\mathbf{A}$} \\
\hline $\begin{array}{l}\lambda_{\max } \\
(\mathrm{nm})\end{array}$ & $\begin{array}{c}\varepsilon \\
\left(\times 10^{4} \mathrm{M}^{-1} \mathrm{~cm}^{-1}\right) \\
\end{array}$ & $\begin{array}{c}\lambda_{\text {em }} \\
(\mathrm{nm})\end{array}$ & $\Phi_{\mathrm{F}}$ & $\begin{array}{c}\tau_{\mathrm{F}} \\
\text { (ns) }\end{array}$ & $\begin{array}{l}\text { Brightness } \\
\left(\varepsilon \times \Phi_{\mathrm{F}}\right)\end{array}$ \\
\hline \multicolumn{6}{|l|}{ Cy5 } \\
\hline 647 & $27.03 \pm 0.8$ & 665 & 0.26 & 1.09 & 70,200 \\
\hline \multicolumn{6}{|c|}{ Cy5B-disulfo } \\
\hline 669 & $19.3 \pm 2.4$ & 684 & 0.45 & 1.89 & 86,850 \\
\hline \multicolumn{6}{|c|}{ Cy5B-trisulfo } \\
\hline 669 & $24.1 \pm 1.9$ & 685 & 0.40 & 1.85 & 96,400 \\
\hline \multicolumn{6}{|l|}{ B } \\
\hline \multicolumn{2}{|c|}{ Construct } & $\Phi_{F}$ & $\frac{\Phi_{F}-\text { labeled }}{\Phi_{F}-\text { free dye }}$ & $\begin{array}{c}\tau_{F} \\
(n s) \\
\end{array}$ & $\begin{array}{c}\tau_{F} \text { - labeled } \\
\tau_{F}-\text { free dye }\end{array}$ \\
\hline \multicolumn{6}{|l|}{ DOL-1 } \\
\hline \multicolumn{2}{|c|}{ Cy5-mAb } & 0.21 & 0.81 & 1.53 & 1.41 \\
\hline \multicolumn{2}{|c|}{ Cy5B-disulfo- mAb } & 0.38 & 0.84 & 2.08 & 1.10 \\
\hline \multicolumn{2}{|c|}{ Cy5B-trisulfo- mAb } & 0.41 & 1.03 & 2.13 & 1.15 \\
\hline \multicolumn{6}{|l|}{ DOL-3 } \\
\hline \multicolumn{2}{|c|}{ Cy5-mAb } & 0.11 & 0.42 & 1.22 & 1.12 \\
\hline \multicolumn{2}{|c|}{ Cy5B-disulfo- mAb } & 0.31 & 0.69 & 1.79 & 0.95 \\
\hline \multicolumn{2}{|c|}{ Cy5B-trisulfo- mAb } & 0.39 & 0.98 & 2.21 & 1.19 \\
\hline
\end{tabular}

resulting conjugates. Overall, these studies illustrate that cyanine conformational restraint, when combined with optimized sulfonation, can provide fluorophore-antibody conjugates with excellent optical properties.

To investigate the impact of conformational restraint on the photophysical properties of DNA-conjugates, we immobilized single dyes on a glass slide via DNA origami nanostructures and employed fluorescence correlation spectroscopy (FCS) as an analytical tool to investigate short-lived transient species. We used the well-established ROXS buffer $^{[9]}$ and appended both probes, Cy5B-disulfo and AF647 to DNA-origami structures (Figure 2B, inset). AF647 exhibits a dark state in the microsecond time scale, consistent with trans-to-cis isomerization reported previously. ${ }^{[10]}$ By contrast, Cy5B-disulfo provides a stable fluorescence signal both in the millisecond and microsecond time regime. Of note, the residual autocorrelation term in the Cy5B-disulfo can be attributed to fast blinking not completely removed by $\mathrm{ROXS}^{[9]}$ and is present in both samples.

The lack of the photoisomerization process provides a "cleaner" fluorescent signal on up to the microsecond time range and increases the overall brightness of the molecule since the dark cis-state can no longer be populated. This becomes particularly relevant when excitation rates are very high, as in plasmonic nanostructures. ${ }^{[1]}$ To examine if the rigidified Cy5B-disulfo derivative provides advantages under high-duty cycle imaging conditions, we placed the fluorophores in the plasmonic hotspot formed by two $100 \mathrm{~nm}$ gold nanoparticles using DNA origami nanoantennas (Figure $2 \mathrm{~A})^{[12]}$ and extracted spot-wise fluorescence intensity values from confocal microscopy scans. When plotted as a function of normalized excitation intensity, Cy5B-disulfo is significantly brighter than AF647 at all excitation intensities. While both samples show a degree of saturation, ${ }^{[13]}$ Cy5Bdisulfo starts saturating only at much higher excitation intensities (Figure 2C). At $1 \mu \mathrm{W}$ excitation intensity, Cy5Bdisulfo is more than 3-fold brighter than AF647. These findings indicate that these probes are well-suited for experiments requiring high temporal resolution (i.e., high excitation intensities). ${ }^{[14]}$

Diffusion-based single-molecule FRET (smFRET) ${ }^{[15]}$ has become a popular method to study structural changes and dynamics of biomacromolecules in aqueous solution. However, photoinduced dye blinking, e.g., caused by triplet-state formation, cis-trans photoisomerization, or irreversible photobleaching can severely impact the information accessible via this technique. ${ }^{[16]}$ These artifacts can lead to a reduction of the quality of FRET efficiency histograms or limit temporal resolution and distance accuracy. We evaluated the performance of Cy5B-disulfo and Cy5B-trisulfo in diffusion-based smFRET experiments using microsecond alternating excitation ( $\mu$ sALEX) (Figure 3, Figure S2-S4). ${ }^{[1]}$ The conformationally restrained sulfonated cyanine dyes were benchmarked against commonly-used dye combinations (donor dye AF555 with acceptor AF647 and ATTO647N). ${ }^{[18]}$ The protein model system MalE, a periplasmic component of an ABC transporter, ${ }^{[19]}$ was used as a double-cysteine variant (Thr36Cys-Ser352Cys) ${ }^{[20]}$ that allows studying the conformational changes of MalE via labeling with maleimide fluoro- 

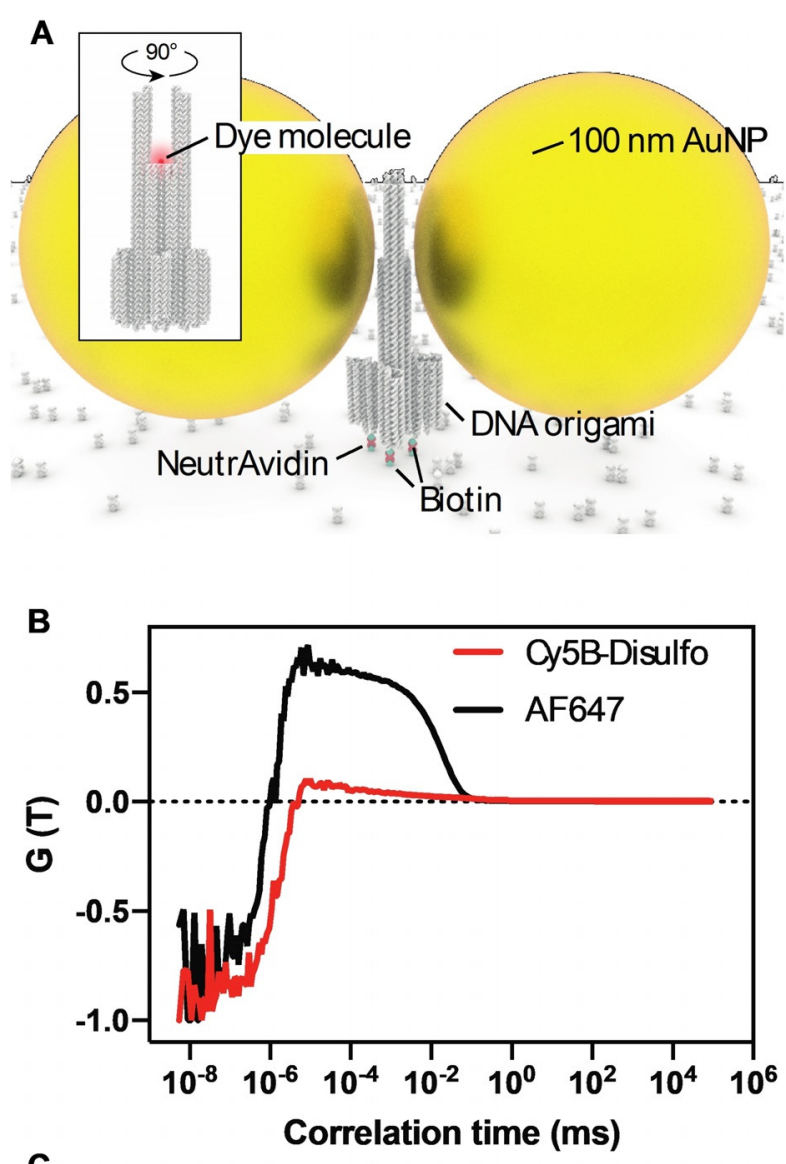

C

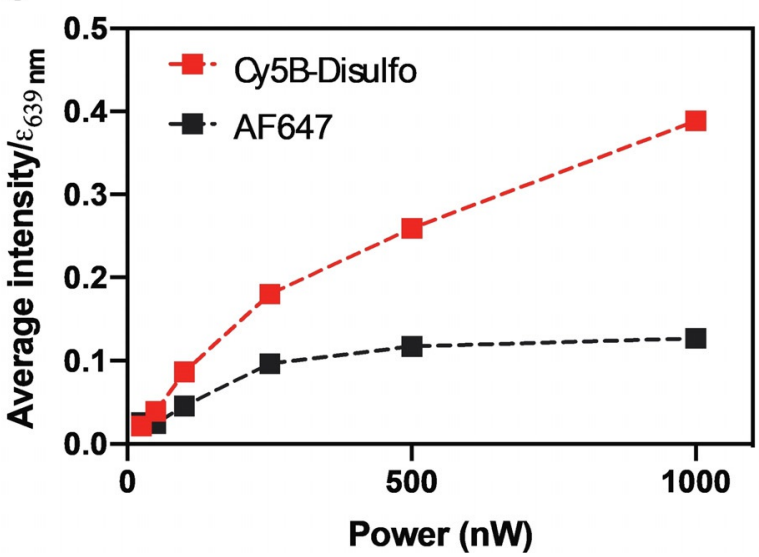

Figure 2. (A) A sketch of DNA origami structure (inset) and full DNA nanoantenna with two $100 \mathrm{~nm}$ Ag NP used to compare photophysical properties of rigidified (Cy5B-disulfo) and non-rigidified (AF647) cyanine dyes. (B) Average autocorrelation of fluorescence signal obtained from single Cy5B-disulfo and AF647 molecules (33 and 37 molecules, respectively) immobilized on a glass slide via DNA origami nanostructures and excited at $639 \mathrm{~nm}$ in the presence of ROXS and in the absence of oxygen. (C) Average intensity of single Cy5B-disulfo and AF647 in the hotspot of DNA nanoantennas obtained at increasing excitation intensities. The fluorescence intensities of single molecules were normalized to the extinction coefficient at the excitation wavelength $(639 \mathrm{~nm})$.

phores (Figure 3 A,B, Figure S2, see SI for the synthesis of the maleimide derivatives and confirmation of double labeling by SEC analysis). ${ }^{[15 b, 16,20 a]}$
The labeling positions on MalE were chosen such that the apo conformation (in the absence of maltose) displays lower FRET efficiency in comparison to the holo state $(100 \mu \mathrm{M}$ maltose) of the protein (Figure $3 \mathrm{~A}$ ). $\mu$ sALEX analysis of such a protein preparation (Figure $3 \mathrm{~B}$, selected fraction marked in grey) is expected to show multiple species in the twodimensional histogram (Figure 3C) of apparent FRET $E^{*}$ ( $x$-axis) and stoichiometry $S^{*}$ (y-axis). While donor-only (D only, high $S^{*}$, low $E^{*}$ ) and acceptor-only subpopulations (A. only, low $S$, intermediate to high $E^{*}$ ) are well separated from the donor-acceptor-containing proteins (DA), photophysical artifacts such as blinking or bleaching manifest as bridges between the latter (denoted as donor and acceptor photophysics). ${ }^{[16,21]}$ Such unwanted photophysical behavior of either donor or acceptor dye can thus alter the "true" mean FRET-efficiency value of an observed species or broaden populations in the FRET efficiency histograms. While broadening is often interpreted as fast (sub-millisecond) structural dynamics, a change of FRET efficiency caused by photophysical effects might be assigned incorrectly to a longer distance.

The $\mu$ sALEX histograms of AF555/Cy5B-trisulfo show a large $(\approx 30 \%)$ donor-acceptor population, where distinct FRET efficiencies are seen for the unliganded apo and liganded holo form (Figure 3D). The FRET efficiency changes between 0.72 in the apo state and 0.90 in the holo state (in presence of $100 \mu \mathrm{M}$ maltose). Both distributions reveal minimal bridging artifacts connecting the FRET population with the donor- and acceptor-only populations. The increasing FRET efficiency upon ligand addition can be interpreted such that the protein adopts the (low FRET) open conformation in the absence of ligand, which is altered into the closed-liganded conformation when $100 \mu \mathrm{M}$ maltose were added. Hence, the assay was validated by reproducing the earlier results and structural predictions. ${ }^{[20 \mathrm{a}]}$ In fact, all acceptor-AF555 combinations visualized the expected conformational changes upon addition of maltose (Figure $4 \mathrm{~A}$ ). Due to the spectral similarity between the acceptor dyes, all dye-pairs showed similar, yet not identical, mean FRET efficiencies and width with good histogram quality (that is, little bridge artifacts due to donor or acceptor photophysics, see also Figure S3 for the 2D $E^{*}-S^{*}$ histograms). Indeed, the cleanest histogram was obtained for Cy5B-trisulfo as acceptor dye (Figure 4A, bottom row) lacking any bleaching trail at FRET values lower than the peak. For all dye-combinations, we also found the expected response of the FRET assays upon addition of $1.5 \mu \mathrm{M}$ maltose (fraction closed of 0.3-0.4), a concentration close to the dissociation constant $\mathrm{K}_{\mathrm{d}}$ for the protein-substrate interaction (Figure S3). ${ }^{[20 a]}$

To quantitatively benchmark the dyes against each other, we compared their acceptor photon count rates at $60 \mu \mathrm{W}$ green and $25 \mu \mathrm{W}$ red laser excitation intensity. Strikingly, we found a maltose dependent fluorophore brightness, where all dyes were similarly bright in the holo state, yet the Cy5B dyes were slightly less bright (by $5-10 \mathrm{kHz}$ ) in comparison with AF647 and ATTO647N in the apo state (Figure 4B). This observation suggests that less FRET bursts can be retained for AF555-Cy5B-monosulfo and AF555-Cy5B-trisulfo compared to the other two fluorophore pairs (Figure 4C). Never- 
A

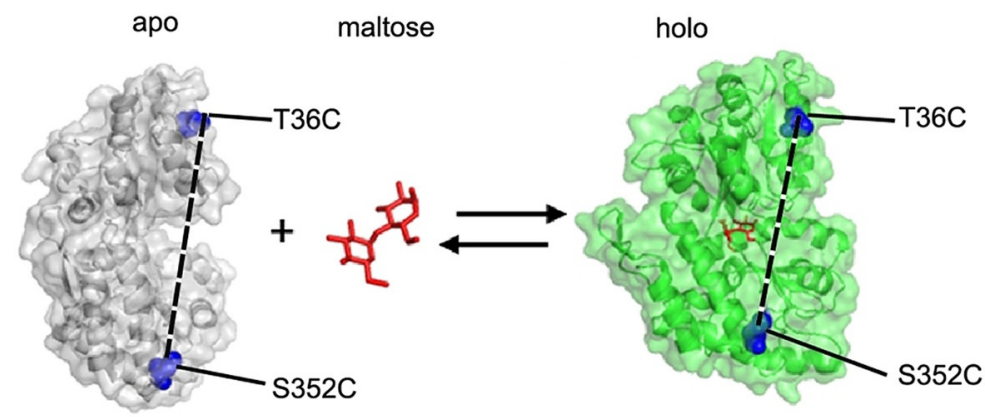

C

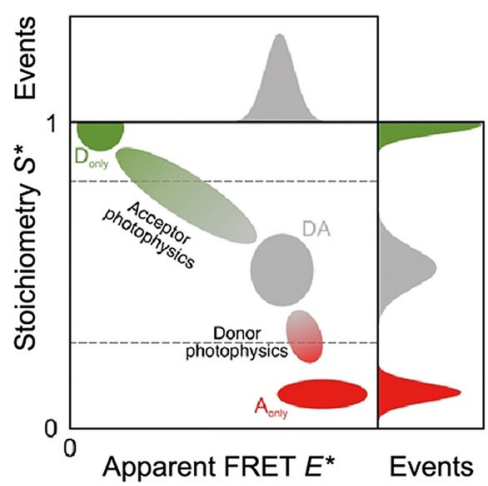

D
B
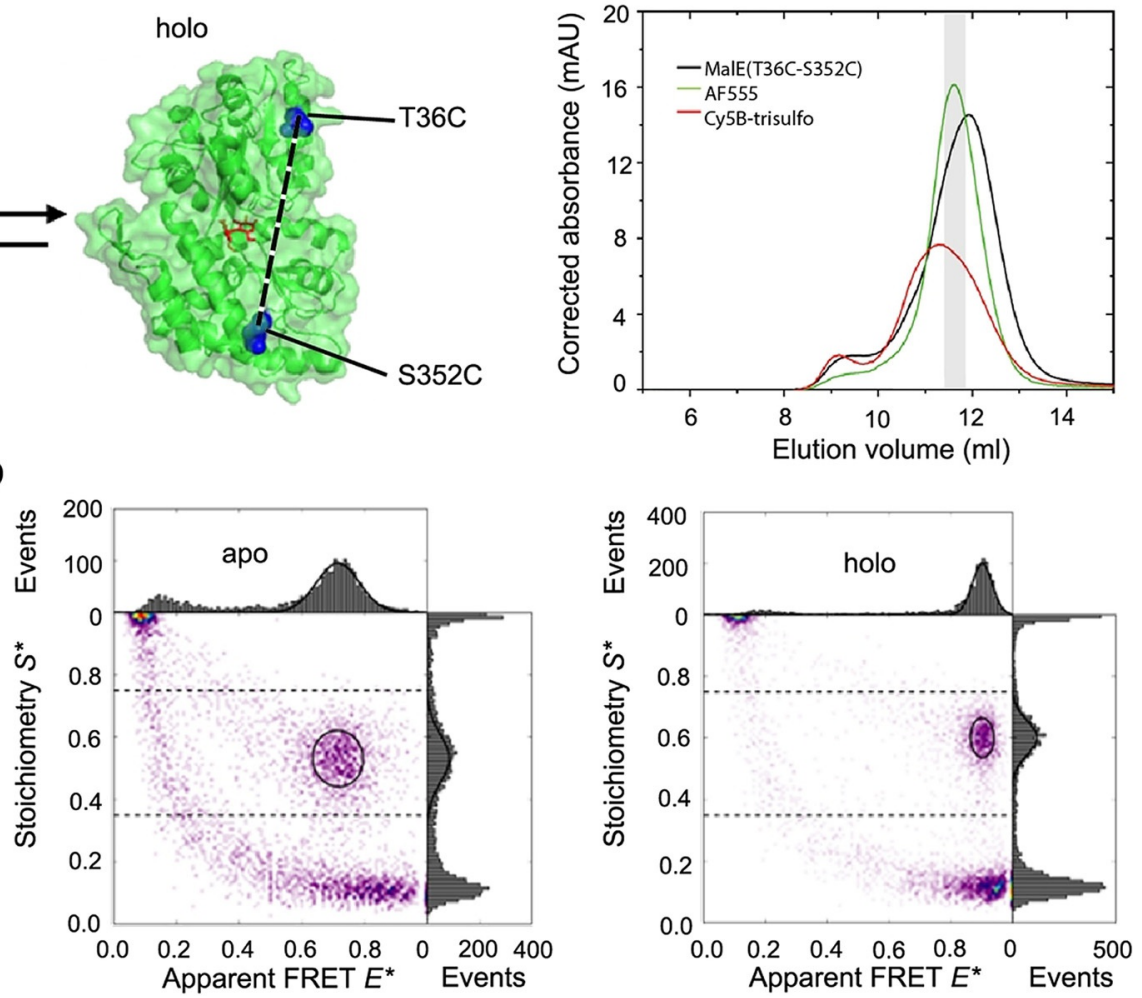

Figure 3. (A) Crystal structure of MalE in apo (grey, pdb file:1omp) and holo (green, pdb file:1anf) where the labeling positions Thr36Cys and Ser352Cys are indicated in blue. (B) Size-exclusion chromatography (SEC) was used to purify the fluorescently labelled protein. The protein absorption was measured at $280 \mathrm{~nm}$ (black curves), donor dye absorption (AF555) at $555 \mathrm{~nm}$, and acceptor dye absorption (Cy5B) at $683 \mathrm{~nm}$ and Cy5B-trisulfo at $688 \mathrm{~nm}$. For the smFRET measurements, the fraction with the best overlap of protein, donor, and acceptor absorption was used, as indicated in grey. (C) Schematic depiction of an $E^{*}-S *$ histogram obtained by $\mu$ SALEX. Using $\mu$ sALEX, the stoichiometry $S^{*}$ can be used to separate donor only $\left(D_{\text {only }}\right)$, acceptor only $\left(A_{\text {only }}\right)$, and the FRET species with both donor and acceptor fluorophore (DA). Bridge artifacts caused by unwanted donor or acceptor photophysics (blinking and/or bleaching) can result in artificial broadening of the FRET population or a shift of the extracted mean apparent FRET efficiency. (D) Typical $\mu$ SALEX-based E*-S* histograms of the MalE double-cysteine mutant labeled with AF555 and Cy5B.

theless, the Cy5B derivatives showed a unique advantage over both AF647 and ATTO647N. Due to the absence of trans-cis isomerization (in comparison to AF647) we found in a laser power dependence in the experiments of the apo state (Figure 4D, Figure S4) that the Cy5B dye had strongly reduced bridge artifacts caused by the acceptor dye (see also Figure $3 \mathrm{C}$ ) and was able to retain the mean $E^{*}$ position and width $\sigma_{\mathrm{E}}$ of the population over the entire range of excitation powers studied (Figure 4D). ${ }^{[16]}$ Also, Cy5B-trisulfo had only a small change of mean peak position, yet substantial change of the population width, which was similar to ATTO647N.

Overall, these observations establish Cy5B and Cy5Btrisulfo as useful alternatives to AF647 and ATTO647N in smFRET experiments, particular to reduce blinking and bleaching artefacts for maintaining correct mean FRET efficiencies. Additionally, the Cy5B dyes behave very well at higher laser powers and with the observed photon output, they are also suitable in cases where high temporal resolution is required. We should mention that the observed fluorophore performance may differ significantly for other biomolecular targets. Thus, alternative protein systems or nucleic acid targets should be tested in smFRET studies with Cy5B derivatives as acceptor dye (in combination with green absorbing donors) or as a donor dye for near infrared acceptors. We also suggest that the Cy5B derivatives might be particularly useful for (smFRET) assays, where interactions of nucleic acids and proteins are monitored, since conventional cyanine dyes often show (unwanted) environmentdependent fluorescence lifetimes ${ }^{[22]}$ which can be minimized for rigidized derivatives of $\mathrm{Cy} 3 \mathrm{~B}$ or $\mathrm{Cy} 5 \mathrm{~B} \cdot{ }^{[23]}$

Having established the utility of these probes for in vitro single-molecule applications, we evaluated them for cellular applications. Multiplexing and multidimensional microscopy of different target molecules in parallel in the red spectral range remains challenging. To enable multiplexing, fluorescence characteristics have to clearly differ for unequivocal identification while they should be efficiently excitable with the same laser to reduce chromatic aberrations and experimental complexity. Therefore, we asked if the difference in fluorescence lifetimes of the sulfonated Cy5B and Cy5 antibody conjugates could be used for two-color fluorescence lifetime imaging microscopy (FLIM). ${ }^{[25]}$ We performed FLIM measurements of Cy5 immunolabeled $\beta$-tubulin and detected an average fluorescence lifetime of $0.91 \mathrm{~ns}$ (Figure 5), whereas cells immunolabeled solely for clathrin with Cy5B-trisulfo showed a fluorescence lifetime of $\approx 1.75 \mathrm{~ns}$ similar to the 
A

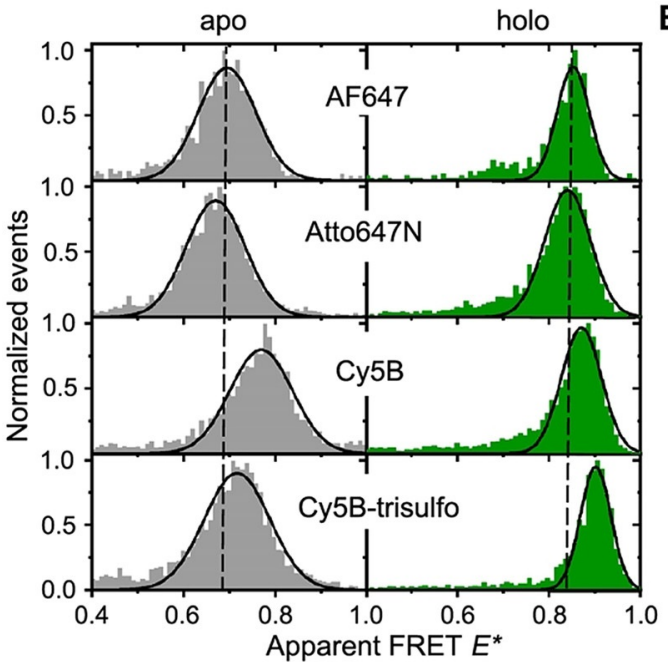

B

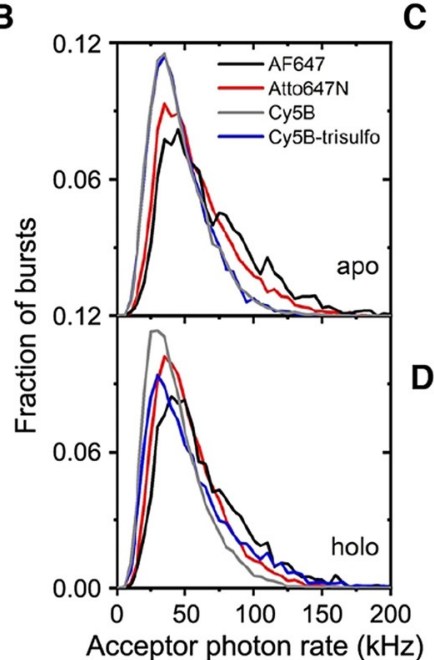

C

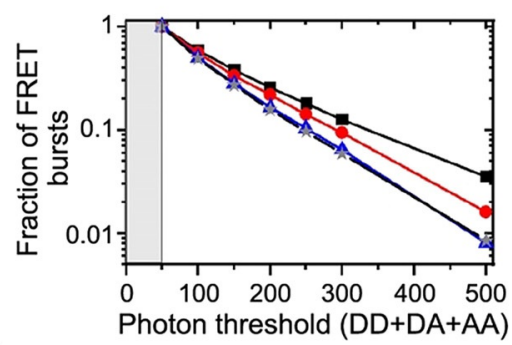

D

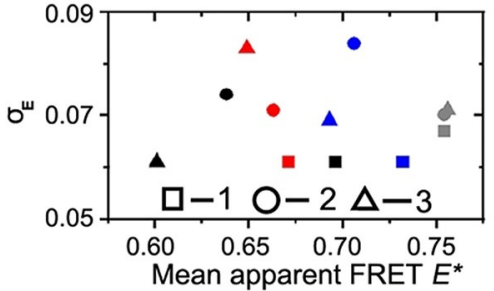

Figure 4. (A) Apparent FRET efficiency E* histograms of MalE obtained from single molecule $\mu$ sALEX FRET experiments. Different FRET acceptor fluorophores as indicated in the Figure panels were combined with the same donor fluorophore (AF555). E* histograms were recorded in the absence (apo) and presence of saturating ligand concentrations of $100 \mu \mathrm{M}$ maltose (holo). Solid lines are the projections of 2D Gaussian fits to the data from which the mean apparent FRET efficiency and width of the histogram was determined (see also panel D). All histograms shown are projections from bursts with a stoichiometry between 0.3 and 0.7. See Figure S3 for full 2D datasets including intermediate ligand concentration. (B) Photon counting histograms ( $\mathrm{PCH}$ ) of the same data sets as in $(\mathrm{A})$ obtained from direct acceptor excitation for the protein in the apo and holo state. Only bursts with an apparent FRET efficiency of 0.4-1 and a stoichiometry between 0.3 and 0.8 were used for the analysis. The acceptor photon count rate was determined by comparison of absolute number of detected photons and the respective burst length. (C) Fraction of bursts with intermediate stoichiometry values (0.3-0.7) where both donor and acceptor dye were present ("FRET bursts") as a function of increasing all photon threshold $(\mathrm{DD}=$ Donor emission after Donor excitation, $\mathrm{DA}=$ Acceptor emission after Donor excitation, $\mathrm{AA}=\mathrm{Acceptor}$ emission after Acceptor excitation). The survival fraction of FRET bursts was normalized to the number of FRET bursts at a photon threshold of 50 . All data were all recorded in the absence of ligand. The same colors were used as in panel B to indicate the different acceptor dyes. (D) Influence of excitation power on the mean apparent FRET efficiency $E^{*}$ and the width of the $\sigma_{\mathrm{E}}$ of the gaussian fit for the four dye combinations (full data sets in Figure S4, same color code as panel B, see SI excitation conditions SI).
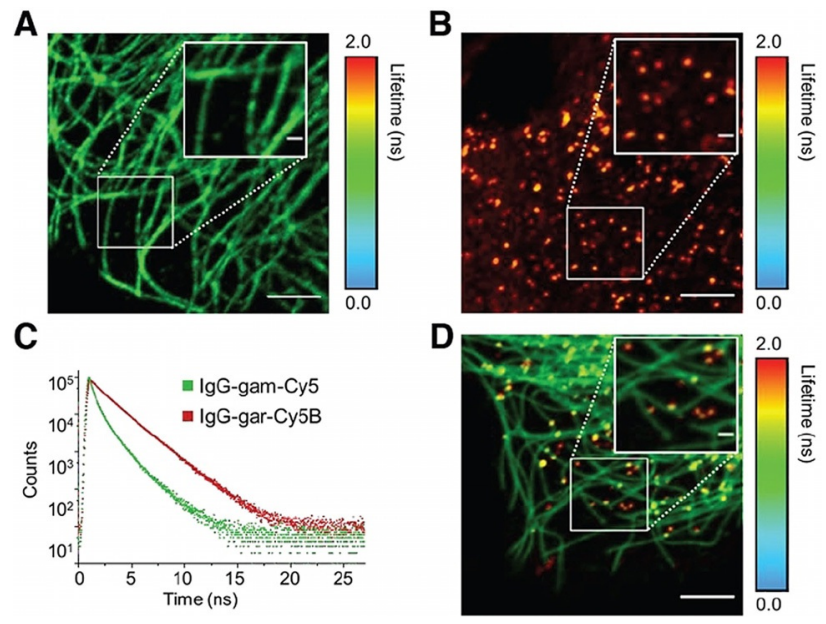

Figure 5. (A) Fluorescence decays of goat anti-rabbit IgG labeled with Cy5B-disulfo (DOL 3.2) and goat anti-mouse IgG labeled with Cy5 (DOL 2) excited at $640 \mathrm{~nm}$. The decays were measured at the emission maxima in PBS, pH 7.4. (B) FLIM image of a COS7 cell immunostained with mouse anti-ß-tubulin primary and goat anti-mouse secondary antibodies labeled with Cy5 (DOL 2). (C) FLIM image of a COS7 cell immunostained with rabbit anti-clathrin primary and goat antirabbit secondary antibodies labeled with Cy5B-disulfo (DOL 3.2).

(D) FLIM composite image of a COS7 cell immunostained with mouse anti-ß-tubulin primary and rabbit anti-clathrin primary and corresponding secondary Cy5 and Cy5B-disulfo labeled antibodies. Scale bars, $5 \mu \mathrm{m}$ and $1 \mu \mathrm{m}$ (insets). values measured in ensemble TCSPC experiments (Table 1). We were able to obtain composite FLIM images of COS7 cells immunolabeled with both dyes based on the different fluorescence lifetimes of $\mathrm{Cy} 5$ and Cy5B-trisulfo labeled antibodies (Figure 5D).

Finally, we evaluated the performance of the new redabsorbing dyes in single-molecule localization microscopy (SMLM) experiments. SMLM with red-absorbing organic dyes are mainly limited to Cy5 and AF647 because of their favorable blinking characteristics in photoswitching buffer ${ }^{[1]}$ $d \mathrm{STORM}^{[26]}$ experiments typically achieve localization precisions of $\approx 10 \mathrm{~nm}$, which translates into $20-30 \mathrm{~nm}$ spatial resolution. ${ }^{[1]}$ We previously reported that these conformationally restricted cyanines do not undergo efficient blinking under conventional high-thiol $d$ STORM conditions. Instead, these probes exhibit improved recovery from $\mathrm{NaBH}_{4}$ reduction, providing high-quality SMLM imaging when appended to a small molecule targeting agent. ${ }^{[3 a, 27]}$

To compare the performance of Cy5 in dSTORM experiments with $\mathrm{Cy} 5 \mathrm{~B}$ in reducing buffer we immunostained $\beta$ tubulin of COS7 cells with Cy5 and Cy5B-trisulfo, as this is a well-established reference structure, to investigate the spatial resolution of super-resolution microscopy methods. Microtubules are hollow tubes with an outer diameter of $25 \mathrm{~nm}$ and $60 \mathrm{~nm}$, respectively, after immunostaining with primary and secondary antibodies. ${ }^{[28]} d$ STORM imaging of Cy5 (Figure 6 A) was performed in standard photoswitching buffer (100 mM MEA, pH 7.5, without enzymatic oxygen 
A
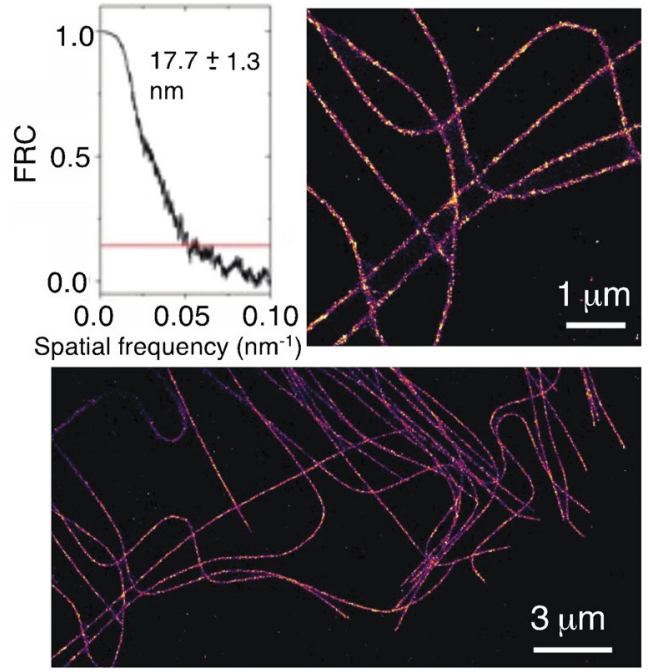

B

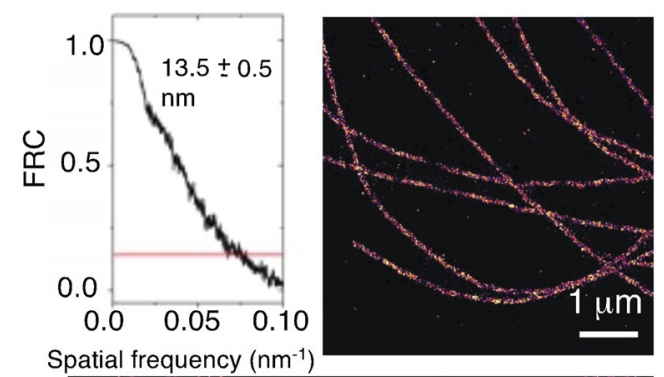

Spatial frequency $\left(\mathrm{nm}^{-1}\right)$

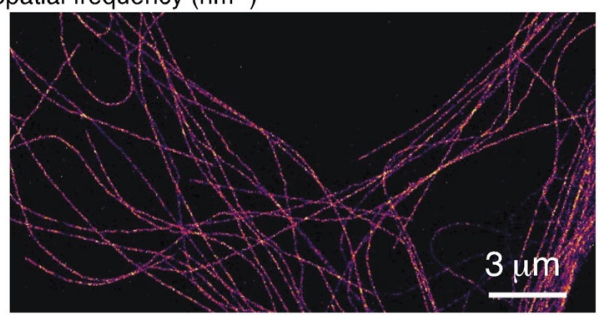

Figure 6. Single-molecule localization microscopy (SMLM) images of microtubules in immunolabeled with Cy5 (A) in $100 \mathrm{mM}$ MEA/PBS $(\mathrm{pH} 7.5)$ and $\mathrm{Cy} 5 \mathrm{~B}$-trisulfo (B) in $0.1 \% \mathrm{NaBH}_{4} / \mathrm{PBS}$ upon irradiation with $640 \mathrm{~nm}\left(2.5 \mathrm{~kW} \mathrm{~cm}^{-2}\right)$ using an integration time of $20 \mathrm{~ms}$ (total acquisition time $10 \mathrm{~min}$ ). Scale bars, $3 \mu \mathrm{m}$ and $1 \mu \mathrm{m}$ (insets). Fourier ring correlation (FRC) analysis ${ }^{[24]}$ were used to estimate the indicated spatial resolutions.

depletion) whereas Cy5B-trisulfo was reduced over $30 \mathrm{~min}$ in $\mathrm{NaBH}_{4} / \mathrm{PBS}$ and measured in the same buffer by TIRF microscopy using solely $640 \mathrm{~nm}$ irradiation (Figure 6B). ${ }^{[6]}$ Removal of residual reductant $\mathrm{NaBH}_{4}$ and imaging in PBS provided a similar image quality (Figure S5). Additional irradiation at $405 \mathrm{~nm}$ did not significantly change the SMLM image quality (Figure S6). For both dyes, Cy5 and Cy5Btrisulfo we could reconstruct high quality super-resolved microtubule images but for Cy5B-trisulfo we detected twice as many photon counts per ON-event $(\approx 1,800)$ as for Cy5 $(\approx 770)$, which translates into higher localization precisions of $6.6 \pm 3.3 \mathrm{~nm}$ (s.d.) for Cy5B-trisulfo and $8.4 \pm 4.4 \mathrm{~nm}$ (s.d.) for Cy5. ${ }^{[29]}$ Fourier ring correlation analysis ${ }^{[24]}$ of microtubule SMLM images corroborated these numbers and provided spatial resolutions of $\approx 13 \mathrm{~nm}$ for Cy5B-trisulfo and $\approx 18 \mathrm{~nm}$ for Cy5. Our data clearly show that Cy5B outperforms classical dSTORM with Cy5 and enables significantly improved spatial resolution. Notably, we also compared samples immunostained with Cy5 and AF647 and see no significant difference in the spatial resolution, despite the somewhat higher aggregation of the Cy5 conjugates (Figure S7). Hence, Cy5B represents a valuable red-absorbing dye with which to apply SMLM with superior spatial resolution and should be consider in future applications. ${ }^{[30]}$

\section{Conclusion}

The development of methods to improve fluorophore photostability and quench reactive and dark states have enabled labeling and monitoring single molecules for up to hundreds of seconds. ${ }^{[10,31]}$ Cyanines have been dyes of choice in numerous types of these single-molecule fluorescence imaging applications. However, when it comes to studying fluorescence processes occurring at faster time scales, the application of these probes is limited by the trans-cis isomerization process, ${ }^{[9]}$ leading to complex blinking dynamics in the microsecond time scale. We previously described the development of constrained variants of cyanines in order to circumvent the depopulation of excited state this isomerization. ${ }^{[3 a, b, c]}$ While conjugation with the first generation of constrained pentamethine cyanines was not possible, here we report the optimized synthesis and evaluation of next generation conformationally restricted pentamethine cyanines.

These new probes enabled us to ask the question, in what context does reduced trans-to-cis isomerization translate to improved imaging performance? FCS studies revealed that in the presence of photostabilization additives, Cy5B-disulfo provides a stable fluorescence signal in the millisecond and microsecond time scale. Comparing the performance of Cy5B-disulfo to non-rigidified AF647 under high excitation intensities in the hotspots of DNA origami nanoantennas demonstrated significantly improved brightness and slower saturation behavior for Cy5B-disulfo. We then extend these observations to smFRET studies, an application where brightness and dye stability is critical. In smFRET experiments dye blinking and bleaching can impact the overall temporal resolution of the imaging experiment and introduce an artificial shift of the extracted FRET efficiency and hence the determined distance between donor and acceptor. While not improving photon-output relative to AF647 and ATTO647N, the Cy5B probes reduce blinking and bleaching artefacts in combination with narrower FRET peaks, particularly when higher laser powers are used. This makes these dyes suitable for, for example, extracting accurate distances from complex biomolecular systems where increased laser powers are required or in cases where a higher temporal resolution is needed. We also demonstrate that the reduced isomerization extends the fluorescence lifetime sufficiently such that constrained and non-constrained cyanines can be used in tandem for multi-color FLIM imaging. Lastly, these new probes are employed for mAb-targeted SMLM, where the unique capacity of these probes to efficiently recover from $\mathrm{NaBH}_{4}$ translates to high photon output and improved 
resolution imaging relative to conventional cyanines in dSTORM.

Broadly, these studies provide a new set of well-characterized far-red probes with utility in demanding fluorescencebased applications. Going forward, these results suggest the use of constrained pentamethine cyanines for live cell SMLM, as imaging can be performed without additives or deoxygenation. Additionally, emerging labeling strategies that exploit their enhanced brightness is of significant interest. ${ }^{[32]} \mathrm{We}$ also believe that these $\mathrm{Cy} 5 \mathrm{~B}$ derivatives might be particularly well suited to smFRET applications looking at interactions of nucleic acids and proteins. This is because conventional cyanines often manifest undesired environment-dependent fluorescence lifetimes, ${ }^{[22]}$ which are minimized for rigidized variants. ${ }^{[23]}$ It is also feasible that direct coupling to photostabilization additives may provide further improvements in the stability of fluorescence signals, as well as photon budgets. ${ }^{[31 \mathrm{~b}, \mathrm{~d}, 33]}$ We note that these restrained cyanines do not undergo photoconversion to the corresponding truncated trimethine cyanine products, which may be advantageous in certain settings. ${ }^{[34]}$ We also anticipate that these probes can be used for DNA point accumulation for imaging in nanoscale topography (DNA-PAINT), an approach that will benefit from higher photon output and can be limited by blinking issues. Efforts to realize these goals will be reported in due course.

\section{Acknowledgements}

R.M, S.S.M., M.P.L., V.B., and M.J.S. were supported by the Intramural Research Program of the National Institutes of Health, National Cancer Institute, Center for Cancer Research. Dr. Joseph Barchi, NCI-CCR, is thanked NMR assistance and Dr. James Kelley, NCI-CCR, for mass spectrometric analysis. The Biophysics Resource, CCR is acknowledged for use of instrumentation. M.M., D.A.H., G.B. and M.S. thank the European Regional Development Fund (EFRE project "Center for Personalized Molecular Immunotherapy") and the Deutsche Forschungsgemeinschaft (SFB/TR 240, project number 374031971, A07) for financial support. Z.H. is grateful for a CSC PhD scholarship and N.Z. thanks the Alexander von Humboldt foundation for a postdoctoral fellowship. Work in the lab of T.C. was funded by the European Commission (ERC-StG No. 638536-SM-IMPORT). VG acknowledges the support from European Union's Horizon 2020 research and innovation program under the Marie Skłodowska-Curie actions (grant agreement No. 840741) and the support by Humboldt Research Fellowships from the Alexander von Humboldt Foundation. P.T. thanks the Deutsche Forschungsgemeinschaft (excellence cluster e-conversion under Germany's Excellence Strategy-EXC2089/1-390776260; Project-ID 201269156SFB1032). Open Access funding enabled and organized by Projekt DEAL.

\section{Conflict of Interest}

A patent describing these compounds was submitted.

Keywords: conformational restriction - cyanine dyes . DNA nanotechnology · fluorescent dyes . single-molecule fluorescence spectroscopy super-resolution microscopy

[1] M. Sauer, M. Heilemann, Chem. Rev. 2017, 117, 7478-7509.

[2] a) A. P. Gorka, R. R. Nani, M. J. Schnermann, Acc. Chem. Res. 2018, 51, 3226-3235; b) A. P. Gorka, R. R. Nani, M. J. Schnermann, Org. Biomol. Chem. 2015, 13, 7584-7598; c) M. Levitus, S. Ranjit, $O$. Rev. Biophys. 2011, 44, $123-151$; d) R. B. Mujumdar, L. A. Ernst, S. R. Mujumdar, C. J. Lewis, A. S. Waggoner, Bioconjugate Chem. 1993, 4,105-111.

[3] a) M. S. Michie, R. Gotz, C. Franke, M. Bowler, N. Kumari, V. Magidson, M. Levitus, J. Loncarek, M. Sauer, M. J. Schnermann, J. Am. Chem. Soc. 2017, 139, 12406-12409; b) S. S. Matikonda, R. Götz, R. McLaughlin, M. Sauer, M. J. Schnermann, Methods Enzymol. 2020, 641, 225 -244; c) S. S. Matikonda, G. Hammersley, N. Kumari, L. Grabenhorst, V. Glembockyte, P. Tinnefeld, J. Ivanic, M. Levitus, M. J. Schnermann, J. Org. Chem. 2020, 85, $5907-5915$

[4] F. Waldchen, J. Schlegel, R. Gotz, M. Luciano, M. Schnermann, S. Doose, M. Sauer, Nat. Commun. 2020, 11, 887 .

[5] a) M. P. Luciano, S. N. Crooke, S. Nourian, I. Dingle, R. R. Nani, G. Kline, N. L. Patel, C. M. Robinson, S. Difilippantonio, J. D. Kalen, M. G. Finn, M. J. Schnermann, ACS Chem. Biol. 2019, 14 , $934-940 ;$ b) K. Sato, T. Nagaya, Y. Nakamura, T. Harada, R. R. Nani, J. B. Shaum, A. P. Gorka, I. Kim, C. H. Paik, P. L. Choyke, M. J. Schnermann, H. Kobayashi, Mol. Pharm. 2015, 12, $3303-$ 3311; c) S. M. Usama, E. R. Thapaliya, M. P. Luciano, M. J. Schnermann, Curr. Opin. Chem. Biol. 2021, 63, 38-45.

[6] a) S. J. Mason, S. Balasubramanian, Org. Lett. 2002, 4, 4261 4264; b) S. J. Mason, J. L. Hake, J. Nairne, W. J. Cummins, S. Balasubramanian, J. Org. Chem. 2005, 70, 2939-2949; c) D. S. Pisoni, L. Todeschini, A. C. Borges, C. L. Petzhold, F. S. Rodembusch, L. F. Campo, J. Org. Chem. 2014, 79, 5511-5520; d) N. Wolf, L. Kersting, C. Herok, C. Mihm, J. Seibel, J. Org. Chem. 2020, 85, $9751-9760$.

[7] B. J. Harvey, M. Levitus, J. Fluoresc. 2009, 19, 443.

[8] a) H. J. Gruber, C. D. Hahn, G. Kada, C. K. Riener, G. S. Harms, W. Ahrer, T. G. Dax, H.-G. Knaus, Bioconjugate Chem. 2000, 11, $696-704$; b) J. E. Berlier, A. Rothe, G. Buller, J. Bradford, D. R. Gray, B. J. Filanoski, W. G. Telford, S. Yue, J. Liu, C. Y. Cheung, W. Chang, J. D. Hirsch, J. M. Beechem, R. P. Haugland, R. P. Haugland, J. Histochem. Cytochem. 2003, 51, 1699-1712.

[9] J. Vogelsang, R. Kasper, C. Steinhauer, B. Person, M. Heilemann, M. Sauer, P. Tinnefeld, Angew. Chem. Int. Ed. 2008, 47, 5465-5469; Angew. Chem. 2008, 120, 5545-5550.

[10] J. Widengren, P. Schwille, J. Phys. Chem. A 2000, 104, 64166428.

[11] P. Anger, P. Bharadwaj, L. Novotny, Phys. Rev. Lett. 2006, 96, 113002.

[12] a) G. P. Acuna, F. M. Moller, P. Holzmeister, S. Beater, B. Lalkens, P. Tinnefeld, Science 2012, 338, 506-510; b) K. Trofymchuk, V. Glembockyte, L. Grabenhorst, F. Steiner, C. Vietz, C. Close, M. Pfeiffer, L. Richter, M. L. Schütte, F. Selbach, R. Yaadav, J. Zähringer, Q. Wei, A. Ozcan, B. Lalkens, G. P. Acuna, P. Tinnefeld, Nat. Commun. 2021, 12, 950.

[13] L. Grabenhorst, K. Trofymchuk, F. Steiner, V. Glembockyte, P. Tinnefeld, Methods Appl. Fluoresc. 2020, 8, 024003.

[14] H. S. Chung, W. A. Eaton, Curr. Opin. Struct. Biol. 2018, 48, $30-$ 39. 
[15] a) B. Hellenkamp, S. Schmid, O. Doroshenko, O. Opanasyuk, R. Kühnemuth, S. R. Adariani, B. Ambrose, M. Aznauryan, A Barth, V. Birkedal, Nat. Methods 2018, 15, 669-676; b) E. Lerner, A. Barth, J. Hendrix, B. Ambrose, V. Birkedal, S. C. Blanchard, R. Borner, H. Sung Chung, T. Cordes, T. D. Craggs, A. A. Deniz, J. Diao, J. Fei, R. L. Gonzalez, I. V. Gopich, T. Ha C. A. Hanke, G. Haran, N. S. Hatzakis, S. Hohng, S. C. Hong, T. Hugel, A. Ingargiola, C. Joo, A. N. Kapanidis, H. D. Kim, T. Laurence, N. K. Lee, T. H. Lee, E. A. Lemke, E. Margeat, J. Michaelis, X. Michalet, S. Myong, D. Nettels, T. O. Peulen, E. Ploetz, Y. Razvag, N. C. Robb, B. Schuler, H. Soleimaninejad, C. Tang, R. Vafabakhsh, D. C. Lamb, C. A. Seidel, S. Weiss, eLife 2021, 10, e60416; c) E. Lerner, T. Cordes, A. Ingargiola, Y. Alhadid, S. Chung, X. Michalet, S. Weiss, Science 2018, 359, 6373.

[16] X. Kong, E. Nir, K. Hamadani, S. Weiss, J. Am. Chem. Soc. 2007, $129,4643-4654$.

[17] a) A. N. Kapanidis, N. K. Lee, T. A. Laurence, S. Doose, E. Margeat, S. Weiss, Proc. Natl. Acad. Sci. USA 2004, 101, $8936-$ 8941; b) J. Hohlbein, T. D. Craggs, T. Cordes, Chem. Soc. Rev. 2014, 43, 1156-1171.

[18] C. Gebhardt, M. Lehmann, M. M. Reif, M. Zacharias, G. Gemmecker, T. Cordes, ChemPhysChem 2021, 22, 1566-1583.

[19] W. Boos, H. Shuman, Microbiol. Mol. Biol. Rev. 1998, 62, $204-$ 229.

[20] a) M. de Boer, G. Gouridis, R. Vietrov, S. L. Begg, G. K. Schuurman-Wolters, F. Husada, N. Eleftheriadis, B. Poolman, C. A. McDevitt, T. Cordes, eLife 2019, 8, e44652; b) R. Mächtel, A. Narducci, D. A. Griffith, T. Cordes, C. Orelle, Res. Microbiol. 2019, 170, 321-337.

[21] J. H. van der Velde, J. Oelerich, J. Huang, J. H. Smit, A. Aminian Jazi, S. Galiani, K. Kolmakov, G. Guoridis, C. Eggeling, A. Herrmann, G. Roelfes, T. Cordes, Nat. Commun. 2016, 7, 10144.

[22] a) H. Hwang, H. Kim, S. Myong, Proc. Natl. Acad. Sci. USA 2011, 108, 7414-7418; b) F. Rashid, V. S. Raducanu, M. S. Zaher, M. Tehseen, S. Habuchi, S. M. Hamdan, Nat. Commun. 2019, 10, 2104.

[23] a) E. Ploetz, E. Lerner, F. Husada, M. Roelfs, S. Chung, J. Hohlbein, S. Weiss, T. Cordes, Sci. Rep. 2016, 6, 33257; b) E. Lerner, E. Ploetz, J. Hohlbein, T. Cordes, S. Weiss, J. Phys. Chem. B 2016, 120, 6401-6410.

[24] R. P. Nieuwenhuizen, K. A. Lidke, M. Bates, D. L. Puig, D. Grunwald, S. Stallinga, B. Rieger, Nat. Methods 2013, 10, $557-$ 562.

[25] a) P. I. Bastiaens, A. Squire, Trends Cell Biol. 1999, 9, 48-52; b) T. Niehörster, A. Loschberger, I. Gregor, B. Kramer, H. J. Rahn, M. Patting, F. Koberling, J. Enderlein, M. Sauer, Nat.
Methods 2016, 13, 257-262; c) D. A. Helmerich, G. Beliu, M. Sauer, ACS Nano 2020, 14, 12629-12641.

[26] M. Heilemann, S. van de Linde, M. Schuttpelz, R. Kasper, B. Seefeldt, A. Mukherjee, P. Tinnefeld, M. Sauer, Angew. Chem. Int. Ed. 2008, 47, 6172-6176; Angew. Chem. 2008, 120, 62666271.

[27] Y. Gidi, L. Payne, V. Glembockyte, M. S. Michie, M. J. Schnermann, G. Cosa, J. Am. Chem. Soc. 2020, 142, $12681-12689$.

[28] K. Weber, P. C. Rathke, M. Osborn, Proc. Natl. Acad. Sci. USA 1978, $75,1820-1824$.

[29] K. I. Mortensen, L. S. Churchman, J. A. Spudich, H. Flyvbjerg, Nat. Methods 2010, 7, 377-381.

[30] a) F. Balzarotti, Y. Eilers, K. C. Gwosch, A. H. Gynnå, V. Westphal, F. D. Stefani, J. Elf, S. W. Hell, Science 2017, 355, 606612; b) J. Cnossen, T. Hinsdale, R. Ø. Thorsen, M. Siemons, F. Schueder, R. Jungmann, C. S. Smith, B. Rieger, S. Stallinga, Nat. Methods 2020, 17, 59-63.

[31] a) V. Glembockyte, J. Lin, G. Cosa, J. Phys. Chem. B 2016, 120, 11923-11929; b) Q. Zheng, S. Jockusch, G. G. RodríguezCalero, Z. Zhou, H. Zhao, R. B. Altman, H. D. Abruña, S. C. Blanchard, Photochem. Photobiol. Sci. 2016, 15, 196-203; c) I. Rasnik, S. A. McKinney, T. Ha, Nat. Methods 2006, 3, 891-893; d) R. B. Altman, D. S. Terry, Z. Zhou, Q. Zheng, P. Geggier, R. A. Kolster, Y. Zhao, J. A. Javitch, J. D. Warren, S. C. Blanchard, Nat. Methods 2012, 9, 68-71.

[32] a) G. Beliu, A. J. Kurz, A. C. Kuhlemann, L. Behringer-Pliess, M. Meub, N. Wolf, J. Seibel, Z.-D. Shi, M. Schnermann, J. B. Grimm, Commun. Biol. 2019, 2, 261; b) S. S. Nguyen, J. A. Prescher, Nat. Rev. Chem. 2020, 4, 476-489.

[33] a) M. Isselstein, L. Zhang, V. Glembockyte, O. Brix, G. Cosa, P. Tinnefeld, T. Cordes, J. Phys. Chem. Lett. 2020, 11, 4462-4480; b) P. Tinnefeld, T. Cordes, Nat. Methods 2012, 9, 426-427; c) A. K. Pati, O. El Bakouri, S. Jockusch, Z. Zhou, R. B. Altman, G. A. Fitzgerald, W. B. Asher, D. S. Terry, A. Borgia, M. D. Holsey, J. E. Batchelder, C. Abeywickrama, B. Huddle, D. Rufa, J. A. Javitch, H. Ottosson, S. C. Blanchard, Proc. Natl. Acad. Sci. USA 2020, 117, 24305-24315.

[34] a) D. A. Helmerich, G. Beliu, S. S. Matikonda, M. J. Schnermann, M. Sauer, Nat. Methods 2021, 18, 253-257; b) S. S. Matikonda, D. A. Helmerich, M. Meub, G. Beliu, P. Kollmannsberger, A. Greer, M. Sauer, M. J. Schnermann, ACS Cent. Sci. 2021, 7, $1144-1155$.

Manuscript received: July 21, 2021

Revised manuscript received: September 18, 2021

Accepted manuscript online: October 4, 2021

Version of record online: November 17, 2021 